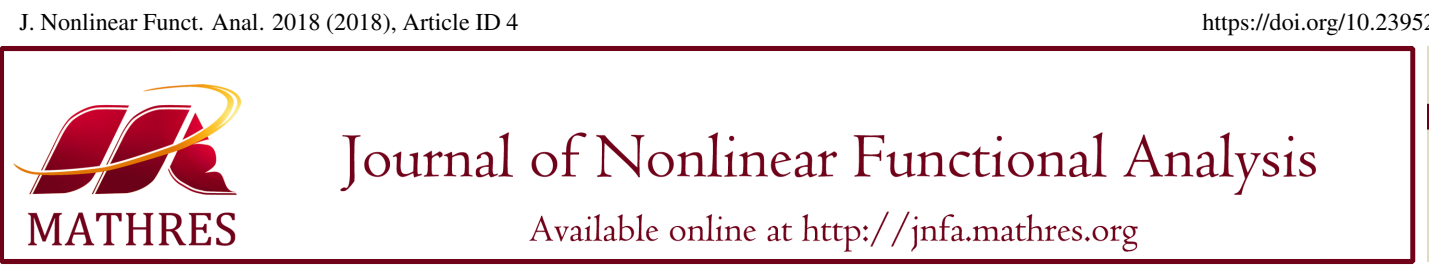

https://doi.org/10.23952/jnfa.2018.4

\title{
QUASI-INTERSECTION PROBLEMS AND FIXED POINT THEOREMS OF L.S.C AND U.S.C MAPPINGS
}

\author{
NGUYEN XUAN TAN ${ }^{1, *}$, NGUYEN BA MINH ${ }^{2}$ \\ ${ }^{1}$ Institute of Mathematics, VAST, 18 Hoang Quoc Viet, 10307 Hanoi, Vietnam \\ ${ }^{2}$ University of Commerce Hanoi, Nanoi, Vietnam
}

\begin{abstract}
We first formulate quasi-intersection problems concerning multivalued mappings and give some sufficient conditions to the existence of their solutions. In particular, we establish several results on the existence of solutions for quasi-equilibrium problems and of fixed points of 1.s.c and u.s.c mappings.

Keywords. Intersection problem; Quasi-equilibrium problem; Upper and lower semi-continuous mapping; Fixed point theorem.
\end{abstract}

2010 Mathematics Subject Classification. 49J27, 49J53, 91B50.

\section{INTRODUCTION}

It is well-known that the intersection problems have closed relations to the fixed point problems and they play an important role in applied mathematics. Many results in this theory become a useful tool to show the existence for solutions and to construct algorithms for finding solutions of many mathematical problems as optimization, variational inequality, complementarity, equilibrium, intersection problems and so on. First, we shortly describe the main development of the fixed theory concerning continuous mappings as follows. In 1912, Brouwer used the combinatorial method to show that a continuous mapping $f$ from a simplex $K \subset R^{n}$ into itself has a fixed point, i.e., there exists a point $\bar{x} \in K$ such that $\bar{x}=f(\bar{x})$. In 1930, Schauder extended this result to the case when $K$ is a nonempty convex compact subset in $R^{n}$. In 1941, Kakutani generalized to the case when $f$ is a upper semi-continuous mapping with nonempty convex and closed values from $K$ to itself in $R^{n}$. In 1952, Fan proved a fixed point theorem of upper semi-continuous mappings with nonempty convex and closed values from a nonempty convex and compact subset $K$ into itself in Hausdorff topological locally convex spaces. In 1968, Browder and Fan obtained a fixed point theorem of multivalued mappings which have lower open sections. Recently, many authors proved fixed point theorems of lower semi-continuous multivalued mappings with

\footnotetext{
${ }^{*}$ Corresponding author.

E-mail addresses: nxtan@math.ac.vn (N.X. Tan), baminhnguyen@tmu.edu.vn (N.B. Minh).

Received July 27, 2017; Accepted January 2, 2018.
}

(C)2018 Journal of Nonlinear Functional Analysis 
nonempty convex closed values using a continuous selection theorem, see, for example, Yannelis and Prabhakar [1], Ben-El-Mechaiekh [2], Horvath [3], Wu [4], Park [5] and many others. In particular, Wu [4] obtained the following result.

Theorem 1.1 ([4]). Let $X$ be a nonempty subset of Hausdorff locally convex topological vector space, let $D$ be a nonempty compact metrizable subset of $X$ and let $T: X \rightarrow 2^{D}$ be a multivalued mapping with the following properties:

(i) $T(x)$ is a nonempty convex closed set for each $x \in X$;

(ii) $T$ is lower semi-continuous.

Then there exists a point $\bar{x} \in D$ such that $\bar{x} \in T(\bar{x})$.

Tan and Hoa [6] generalized the above theorem by the following result.

Theorem $1.2([6])$. Let $D$ be a nonempty convex and compact subset of Hausdorff locally convex topological vector space $X$ and $F: D \rightarrow 2^{D}$ be a lower semi-continuous multivalued mapping with nonempty values. Then there exists a point $\bar{x} \in D$ such that $\bar{x} \in F(\bar{x})$.

Fixed point problems have many connections with variational, equilibrium, and intersection problems. In this paper, we are interested in the existence of solutions of quasi-intersection problems concerning multivalued mappings defined on subsets of Hausdorff locally convex topological vector spaces as follows.

Let $X, Y$ and $Z$ be Hausdorff locally convex topological vector spaces over reals, and let $D \subset X, K \subset Z$ be nonempty subsets. Given multi-valued mappings $P: D \times K \rightarrow 2^{D}, Q: D \times K \rightarrow 2^{K}$ and $G, H: D \times K \rightarrow$ $2^{Y}$, we are interested in the problem of finding $(\bar{x}, \bar{y}) \in D \times K$ such that

$$
\begin{aligned}
& \bar{x} \in P(\bar{x}, \bar{y}), \\
& \bar{y} \in Q(\bar{x}, \bar{y}), \\
& G(\bar{x}, \bar{y}) \cap H(\bar{x}, \bar{y}) \neq \emptyset .
\end{aligned}
$$

This problem is called a quasi-intersection problem (in short, (QIP)), in which the multivalued mappings $P$ and $Q$ are constraint mappings and $G$ and $H$ are utility multivalued mappings that are often determined by equalities and inequalities, or, by inclusions and intersections of other multi-valued mappings, or by general relations in product spaces. The existence of solutions to this problem is studied in $[6,7,8,9,10]$ for the case the multivalued mapping $P$ is continuous, the multivalued mapping $Q$ is u.s.c and the multivalued mapping $F$ is u.s.c. All these mappings $P, Q$ and $F$ need to have nonempty convex and closed values. If we take $H(x, y)=0$ for all $x, y) \in D \times K$, then this problem becomes to a quasi-equilibrium problem. In the case $X=Y$, if we take $H(x, y)=x$ for all $(x, y) \in D \times K$, then this problem becomes to a generalized fixed point problems considered by Tan and Hoa in [6]. As far as we know equilibrium problems as generalizations of variational inequalities and optimization problems, including also optimization-related problems such as fixed point problems, complementarity problems, Nash equilibrium problems, minimax problems and so on. For the last decade, there have been a number of generalizations of these problems to different directions such as quasi-equilibrium problems with constraint sets depending on parameters, quasi-variational and quasi-equilibrium inclusion problems with multi-valued data; see, for examples, in $[6,7,8,9,10]$ and the references therein. Problem (QIP) described above is quite general. It encompasses a large class of problems of applied mathematics 
including quasi-optimization problems, quasi-variational inclusion, quasi-equilibrium problems, quasivariational relation problems, etc. Typical instances of (QIP) are shown in $[6,7,8,10]$ involving upper semi-continuous utility multivalued mappings with nonempty convex closed values.

\section{PRELIMINARIES}

Assume that $X, Y$ are real Hausdorff topological vector spaces and $D \subseteq X$ is a nonempty subset. We consider a multivalued mapping $F: D \rightarrow 2^{Y}$ and $F^{-1}: Y \rightarrow 2^{X}$ defined by $x \in F^{-1}(y)$ if and only if $y \in F(x)$. We recall that

(a) The domain and the graph of $F$ are denoted by

$$
\begin{gathered}
\operatorname{dom} F=\{x \in D \mid F(x) \neq \emptyset\}, \\
\operatorname{Gr}(F)=\{(x, y) \in D \times Y \mid y \in F(x)\},
\end{gathered}
$$

respectively;

(b) $F$ is said to be a closed mapping if the graph $\operatorname{Gr}(F)$ of $F$ is a closed subset in the product space $X \times Y$;

(c) $F$ is said to be a compact mapping if the closure $\overline{F(D)}$ of its range $F(D)$ is a compact set in $Y$;

(d) $F: D \rightarrow 2^{Y}$ is said to be upper semi-continuous (in short, u.s.c) at $\bar{x} \in D$ if for each open set $V$ containing $F(\bar{x})$, there exists an open set $U$ of $\bar{x}$ such that for each $x \in U, F(x) \subset V . \quad F$ is said to be u.s.c on $D$ if it is u.s.c at all $x \in D$;

(e) $F$ is said to be lower semi-continuous ( in short, l.s.c) at $\bar{x} \in D$ if for any open set $V$ with $F(\bar{x}) \cap V \neq$ $\emptyset$, there exists an open set $U$ containing $\bar{x}$ such that for each $x \in U, F(x) \cap V \neq \emptyset$. $F$ is said to be 1. s.c on $D$ if it is 1. s.c at all $x \in D$;

(f) $F$ is said to be continuous on $D$ if it is at the same time u.s.c and l.s.c on $D$;

(g) $F$ is said to have open lower sections if the inverse mapping $F^{-1}$ is open valued, i.e, for all $y \in Y, F^{-1}(y)$ is open in $X$.

The following propositions and theorems are need in this paper. Their proofs can be found in [6].

Proposition 2.1. $F: D \rightarrow 2^{Y}$ is l.s.c at $x \in D, F(x) \neq \emptyset$, if and only if for any $n e t\left\{x_{\alpha}\right\}$ in $D, x_{\alpha} \rightarrow x, y \in$ $F(x)$, there is a net $\left\{y_{\alpha}\right\}$ with $y_{\alpha} \in F\left(x_{\alpha}\right), y_{\alpha} \rightarrow y$.

By $X^{*}$ we denote the dual space of $X$, i.e.,

$$
X^{*}=\{f: X \rightarrow R \mid f \text { is a linear and continuous function }\} .
$$

The pairing $<., .>$ between elements of $p \in X^{*}$ and $x \in X$ is defined by $<p, x>=p(x)$. We have

Proposition 2.2. If $F: D \rightarrow 2^{Y}$ is a l.s.c (u.s.c) multivalued mapping with nonempty values on $D$ and $p \in Y^{*}$, then

i) The function $c_{p}: D \rightarrow R$, defined by $c_{p}(x)=\inf _{v \in F(x)}<p, v>\left(c_{p}(x)=\sup _{v \in F(x)}\langle p, v>\right.$, respectively) is upper semi-continuous on D.

ii) The function $c_{p}: D \rightarrow R$, defined by $c_{p}(x)=\sup _{v \in F(x)}<p, v>\left(c_{p}(x)=\inf _{v \in F(x)}<p, v>\right.$, respectively) is lower semi-continuous on $D$.

Proposition 2.3. Let $F: D \rightarrow 2^{Y}$ be a multivalued mapping with nonempty values on $D$. Then, if $F$ has open lower sections, then $F$ is l.s.c on $D$. 
Remark 2.4. It is easy to give examples proving that a continuous mapping may not have open lower sections.

Conversely, we have the following proposition.

Proposition 2.5. Let $F: D \rightarrow 2^{Y}$ be a l.s.c multivalued mapping with nonempty values on $D$. Then, for any open subset $V$ in $Y$, the multivalued mappings $F_{V}: D \rightarrow 2^{Y}$ defined by $F_{V}(x)=F(x)+V$ has open lower sections.

Remark 2.6. Proposition 2.5 implies that any l.s.c multivalued mapping $F$ can be approximated by a net $\left\{F_{\alpha}\right\}$ of multivalued mappings having open lower sections in the sense: For any net $\left\{x_{\alpha}\right\}$ in $D, x_{\alpha} \rightarrow x$, and $y \in F(x)$, one can find a net $y_{\alpha} \in F_{\alpha}\left(x_{\alpha}\right)$ with $y_{\alpha} \rightarrow y$.

Proposition 2.7. Let $F_{i}: D \rightarrow 2^{Y}, i=1,2$, be a l.s.c (u.s.c) multivalued mapping with nonempty values on $D$. Then, the multivalued mapping $F: D \rightarrow 2^{Y}$ defined by

$$
F(x)=\left(F_{1}+F_{2}\right)(x)=F_{1}(x)+F_{2}(x), x \in D,
$$

is also l.s.c (u.s.c, respectively) on D.

Proposition 2.8. Let $F_{i}: D \rightarrow 2^{Y}, i=1,2$, be multivalued mappings with nonempty values on $D$. Assume that $F_{1}$ is l.s.c and $F_{2}$ has open lower sections. Then, the multivalued mapping $F: D \rightarrow 2^{Y}$ defined by

$$
F(x)=F_{1}(x) \cap F_{2}(x), x \in D,
$$

is l.s.c on D.

Proposition 2.9. Let $F: D \rightarrow 2^{Y}$ be a multivalued mapping with nonempty values on $D$. If $F$ has open lower sections, then the multivalued mapping $\operatorname{coF}: D \rightarrow 2^{Y}$, defined by $(\operatorname{coF})(x)=\operatorname{coF}(x)$, with co $(A)$ denoting the convex hull of $A$, also has open lower sections.

Proposition 2.10. Let $F: D \rightarrow 2^{Y}$ be a l.s.c multivalued mapping with nonempty values on $D$. Then so is the multivalued mapping $\mathrm{coF}: D \rightarrow 2^{Y}$, defined by $(\mathrm{coF})(x)=\operatorname{coF}(x)$.

The following theorems are very important in the proof of the main result in this paper.

Theorem 2.11 ([11]). Let $\left\{V_{\alpha}\right\}_{\alpha \in \Lambda}$ be a an open cover of locally compact Hausdorff space $X$, and let $D \subset X$ be a compact set. Then, there exist continuous functions $\psi_{i}: D \rightarrow R,(i=0,1, \ldots, s)$ such that (i) $0 \leq \psi_{i}(x) \leq 1$;

(ii) $\sum_{i=1}^{s} \psi_{i}(x)=1, \quad \forall x \in D$;

(iii) For any $i \in\{0,1, \ldots, s\}$, there exists $\alpha \in \Lambda$ such that supp $\psi_{i} \subset V_{\alpha}$, where supp $\psi=\{x \in D \mid \psi(x) \neq$ $0\}$.

The system of functions $\left\{\psi_{i}\right\}, i=0,1, \ldots, s$, is said to be a partition of unity corresponding to the open cover $\left\{V_{\alpha}\right\}$.

Theorem 2.12 ([7]). We assume that the following conditions hold:

(i) $D, K$ are nonempty convex compact sets;

(ii) $P: D \times K \rightarrow 2^{D}$ is continuous multivalued mapping with nonempty closed convex values, $Q$ : $D \times K \rightarrow 2^{K}$ is a u.s.c multivalued mapping with nonempty closed convex values; 
(iii) $\phi: K \times D \times D \rightarrow R$ is a continuous function. For any fixed $(y, x) \in K \times D$, the function $\phi(y, x,$.$) :$ $D \rightarrow R$ is quasiconvex and $\phi(y, x, x)=0$.

Then, there exists $(\bar{x}, \bar{y}) \in D \times K$ such that $\bar{x} \in P(\bar{x}, \bar{y}), \bar{y} \in Q(\bar{x}, \bar{y})$ and

$$
\phi(\bar{y}, \bar{x}, z) \geq 0, \quad \forall z \in P(\bar{x}, \bar{y}) .
$$

\section{MAIN RESULTS}

Let $X$ and $Z$ be Hausdorff locally convex topological vector spaces over reals, let $D \subset X, K \subset Z$ be nonempty subsets. Given multi-valued mappings $P: D \times K \rightarrow 2^{D}, Q: D \times K \rightarrow 2^{K}$ and $G, H: D \times K \rightarrow 2^{X}$, we are interested in sufficient conditions for solutions of the problem (GQEP): Find $(\bar{x}, \bar{y}) \in D \times K$ such that

$$
\begin{aligned}
& \bar{x} \in P(\bar{x}, \bar{y}), \\
& \bar{y} \in Q(\bar{x}, \bar{y}), \\
& G \bar{x}, \bar{y}) \cap H(\bar{x}, \bar{y}) \neq \emptyset .
\end{aligned}
$$

Before proving the main results in this section, we recall the following notions. Let $D$ be a subset in $X$ and $x \in D$. The set

$$
T_{D}(x)=\overline{\{\alpha(y-x), y \in D, \alpha \geq 0\}}=\overline{\{\operatorname{cone}(D-x)\}},
$$

is called the tangent cone to the set $D$ at $x$, where cone $M=\{\alpha z, z \in M, \alpha \geq 0\}$.

We now prove the following theorem on the existence for solutions of the above quasi-intersection problem.

Theorem 3.1. We assume that the following conditions hold:

(i) $D, K$ are nonempty convex compact sets;

(ii) $P: D \times K \rightarrow 2^{D}$ is continuous multivalued mapping with nonempty closed convex values, $Q$ : $D \times K \rightarrow 2^{K}$ is a u.s.c multivalued mapping with nonempty closed convex values;

(iii) $G: D \times K \rightarrow 2^{X}$ is a l.s.c multivalued mapping with nonempty values;

(iv) $H: D \times K \rightarrow 2^{X}$ is a u.s.c multivalued mapping with nonempty closed convex values;

(v) For any $(x, y) \in P(x, y) \times Q(x, y), H(x, y)$ is nonempty convex closed and $G(x, y)-H(x, y) \subset$ $T_{P(x, y)}(x)$.

Then there exists $(\bar{x}, \bar{y}) \in D \times K$ such that

1) $\bar{x} \in P(\bar{x}, \bar{y})$

2) $\bar{y} \in Q(\bar{x}, \bar{y})$;

3) $G \bar{x}, \bar{y}) \cap H(\bar{x}, \bar{y}) \neq \emptyset$.

Proof. We set

$$
B=\{(x, y) \in D \times K \mid x \in P(x, y), y \in Q(x, y)\} .
$$

Since the multivalued mapping $T: D \times K \rightarrow 2^{D \times K}$, defined by

$$
T(x, y)=P(x, y) \times Q(x, y),(x, y) \in D \times K,
$$

is upper semi-continuous with nonempty convex and compact values, we conclude from the Ky Fan fixed point theorem that $T$ has a fixed point in $D \times K$. Therefore, $B$ is a nonempty set. The upper semi-continuity and the closedness of values of $T$ imply that $B$ is a closed and then is a compact set. 
Assume that, for any $(x, y) \in B, G(x, y) \cap H(x, y)=\emptyset$. Taking a point $\bar{v} \in G(x, y)$, we find $\bar{v} \notin H(x, y)$. Since $H(x, y)$ is a nonempty closed convex, one has $\bar{v}-H(x, y)$ and $0 \notin(\bar{v}-H(x, y))$. By the HahnBanach theorem, one sees that there exists $p \in X^{*}$ such that

$$
p(\bar{v})-\inf _{w \in H(x, y)} p(w)<0 .
$$

It follows that

$$
\inf _{v \in c o(G(x, y))} p(v)-\inf _{w \in H(x, y)} p(w)<0 .
$$

Further, we define functions $c_{p}{ }^{1}(.,),. c_{p}{ }^{2}(.,):. D \times K \rightarrow R$ by

$$
c_{p}^{1}\left(x^{\prime}, y^{\prime}\right)=\inf _{v \in c o\left(G\left(x^{\prime}, y^{\prime}\right)\right)} p(v),
$$

and

$$
c_{p}^{2}\left(x^{\prime}, y^{\prime}\right)=\inf _{w \in H\left(x^{\prime}, y^{\prime}\right)} p(w) .
$$

By Proposition 2.2, the functions $c_{p}^{1}$ is u.s.c and $c_{p}^{2}$ is 1.s.c on $D \times K$. Therefore, the function $-c^{2}$ is u.s.c and the set

$$
U_{p}(x, y)=\left\{\left(x^{\prime}, y^{\prime}\right) \in D \times K \mid c_{p}{ }^{1}\left(x^{\prime}, y^{\prime}\right)-c_{p}^{2}\left(x^{\prime}, y^{\prime}\right)<0\right\},
$$

is open. Since $(x, y) \in U_{p}(x, y), U_{p}$ is a nonempty open neighborhood of $(x, y)$. Thus, for any $(x, y) \in B$, there is $p \in X^{*}$ such that

$$
U_{p}(x, y)=\left\{\left(x^{\prime}, y^{\prime}\right) \in D \times K \mid c_{p}^{1}\left(x^{\prime}, y^{\prime}\right)-c_{p}^{2}\left(x^{\prime}, y^{\prime}\right)<0\right\}
$$

is nonempty and open and hence $\left\{U_{p}\right\}_{p \in X^{*}}$ is an open cover of $B$. Since $B$ is compact, there exist finite $p_{1}, \ldots, p_{s} \in X^{*}$ such that

$$
B \subseteq \bigcup_{j=1}^{s} U_{p_{j}} .
$$

Further, since $B$ is closed in $D \times K, U_{p_{0}}=D \times K \backslash B$ is open in $D \times K$. Hence $\left\{U_{p_{0}}, U_{p_{1}}, \ldots, U_{p_{s}}\right\}$ is an open cover of the compact set $D \times K$. By Theorem 2.11 in Section 2, there exist continuous functions $\psi_{i}: D \times K \rightarrow R,(i=0,1, \ldots, s)$ such that

(i) $0 \leq \psi_{i}(x, y) \leq 1$;

(ii) $\sum_{i=1}^{s} \psi_{i}(x, y)=1$, for all $(x, y) \in D \times K$;

(iii) For any $i \in\{0,1, \ldots, s\}$, there exists $j(i) \in\{0, \ldots, s\}$ such that supp $\psi_{i} \subset U_{p_{j(i)}}$.

Further, we define the function $\phi: K \times D \times D \rightarrow R$ by

$$
\phi(y, x, t)=\sum_{i=0}^{s} \psi_{i}(x, y) \cdot p_{j(i)}(t-x), \quad \forall t, x \in D, y \in K .
$$

Then, $\phi$ is a continuous function on $K \times D \times D$. Moreover, for any fixed $(x, y) \in D \times K, \phi((y, x,$.$) :$ $D \rightarrow R$ is an affine function and $\phi(y, x, x)=0$ for all $(x, y) \in D \times K$. Therefore, $D, K, P, Q$ and $\phi$ satisfy all conditions of Theorem 2.12 in Section 2. It implies that there is $(\bar{x}, \bar{y}) \in D \times K$ such that $(\bar{x}, \bar{y}) \in$ $P(\bar{x}, \bar{y}) \times Q(\bar{x}, \bar{y})$ and $\phi((\bar{y}, \bar{x}, t) \geq 0$, for all $t \in P(\bar{x}, \bar{y})$. This gives

$$
\sum_{i=0}^{s} \psi_{i}(\bar{x}, \bar{y}) \cdot p_{j(i)}(t-\bar{x}) \geq 0, \quad \forall t \in P(\bar{x}, \bar{y}) .
$$


Setting $p^{*}=\sum_{i=0}^{s} \psi_{i}(\bar{x}, \bar{y}) \cdot p_{j(i)}$, we get from (3.1) that $p^{*}(t-\bar{x}) \geq 0$, for all $t \in P(\bar{x}, \bar{y})$. Hence $p^{*}(u) \geq 0$, for all $u \in T_{P(\bar{x}, \bar{y})}(\bar{x})$. By Assumption $(v), G(\bar{x}, \bar{y})-H(\bar{x}, \bar{y}) \subset T_{P(\bar{x}, \bar{y})}(\bar{x})$, we conclude that

$$
p^{*}(u) \geq 0, \text { for all } u \in G(\bar{x}, \bar{y})-H(\bar{x}, \bar{y}) .
$$

This yields

$$
\inf _{u \in \operatorname{coG}(\bar{x}, \bar{y})-H(\bar{x}, \bar{y})} p^{*}(u) \geq 0 .
$$

Further, put $I(\bar{x}, \bar{y})=\left\{i \in\{0,1, \ldots, s\} \mid \psi_{i}(\bar{x}, \bar{y})>0\right\}$. Since $\psi_{i}(\bar{x}, \bar{y}) \geq 0$ and $\sum_{i=0}^{s} \psi_{i}(\bar{x}, \bar{y})=1$, we deduce $I(\bar{x}, \bar{y}) \neq \emptyset$. So, for any $i \in I(\bar{x}, \bar{y}),(\bar{x}, \bar{y}) \in \operatorname{supp} \psi_{i} \subset U_{p_{j(i)}}$ and

$$
c_{p_{j(i)}}^{1}(\bar{x}, \bar{y})-c_{p_{j(i)}}^{2}(\bar{x}, \bar{y})<0 .
$$

For any $v \in \operatorname{coG}(\bar{x}, \bar{y}), w \in H(\bar{x}, \bar{y})$ we have

$$
\begin{aligned}
p^{*}(v-w) & =\sum_{i}^{s} \psi_{i}(\bar{x}, \bar{y}) \cdot\left(p_{j(i)}(v)-p_{j(i)}(w)\right) \\
& =\sum_{i \in I(\bar{x}, \bar{y})} \psi_{i}(\bar{x}, \bar{y}) \cdot\left(p_{j(i)}(v)-p_{j(i)}(w)\right) \\
& \leq \sum_{i \in I(\bar{x}, \bar{y})} \psi_{i}(\bar{x}, \bar{y}) \max _{i \in I(\bar{x}, \bar{y})}\left(p_{j(i)}(v)-p_{j(i)}(w)\right) \\
& =\max _{i \in I(\bar{x}, \bar{y})}\left(p_{j(i)}(v)-p_{j(i)}(w)\right)
\end{aligned}
$$

Hence,

$$
\begin{aligned}
& \inf _{v-w \in \operatorname{co}(G(\bar{x}, \bar{y}))-H(\bar{x}, \bar{y}))}\left(p^{*}(v-w)\right) \\
& \leq \inf _{v-w \in \operatorname{co} G(\bar{x}, \bar{y})-H(\bar{x}, \bar{y})} \max _{i \in I(\bar{x}, \bar{y})}\left(p_{j(i)}(v)-p_{j(i)}(w)\right)
\end{aligned}
$$

Setting $C=\overline{c o}\left\{p_{j(1)}, \ldots, p_{j(s)}\right\}, E=c o G(\bar{x}, \bar{y})-H(\bar{x}, \bar{y}), f(p, u)=p(v)-p(w)$, and $u=v-w$ and using the weak* topology on $X^{*}$, we can easily verify that all conditions of the Sion's minimax Theorem in [11] are satisfied. Therefore, we obtain

$$
\inf _{u \in \operatorname{coG}(\bar{x}, \bar{y})-H(\bar{x}, \bar{y})} \max _{i \in I(\bar{x}, \bar{y})} p_{j(i)}(u)=\max _{i \in I(\bar{x}, \bar{y})} \inf _{v-w \in \operatorname{coG}(\bar{x}, \bar{y})-H(\bar{x}, \bar{y})}\left(p_{j(i)}(v)-p_{j(i)}(w)\right) .
$$

Taking a fixed $\bar{w} \in H(\bar{x}, \bar{y})$, we have

$$
\begin{aligned}
\inf _{u \in \operatorname{coG}(\bar{x}, \bar{y})-H(\bar{x}, \bar{y})} p_{j(i)}(u) & \leq \inf _{v-\bar{w} \in \operatorname{coG}(\bar{x}, \bar{y})-\bar{w}}\left(p_{j(i)}(v)-p_{j(i)}(\bar{w})\right) \\
& \leq \inf _{v \in \operatorname{coG}(\bar{x}, \bar{y})} p_{j(i)}(v)-\inf _{w \in H(\bar{x}, \bar{y})} p_{j(i)}(w) .
\end{aligned}
$$

Using (3.5), one has

$$
\begin{aligned}
\inf _{u \in c o G(\bar{x}, \bar{y})-H(\bar{x}, \bar{y})} \max _{i \in I(\bar{x}, \bar{y})} p_{j(i)}(u) & =\max _{i \in I(\bar{x}, \bar{y})} \inf _{v-w \in \operatorname{coG}(\bar{x}, \bar{y})-H(\bar{x}, \bar{y})}\left(p_{j(i)}(v)-p_{j(i)}(w)\right) \\
& \leq \max _{i \in I(\bar{x}, \bar{y})}\left\{\inf _{v \in \operatorname{coG}(\bar{x}, \bar{y})} p_{j(i)}(v)-\inf _{w \in H(\bar{x}, \bar{y})} p_{j(i)}(w)\right\} \\
& \leq \max _{i \in I(\bar{x}, \bar{y})}\left\{c_{p_{j(i)}}^{1}(\bar{x}, \bar{y})-c_{p_{j(i)}}^{2}(\bar{x}, \bar{y})\right\}<0 .
\end{aligned}
$$

A combination of (3.4) and (3.6) implies

$$
\inf _{u \in \operatorname{coG}(\bar{x}, \bar{y})-H(\bar{x}, \bar{y})} p^{*}(u) \leq \inf _{v \in \operatorname{coG}(\bar{x}, \bar{y})} p^{*}(v)-\inf _{w \in H(\bar{x}, \bar{y})} p^{*}(w)<0 .
$$


Thus, we have a contradiction to (3.2). This completes the proof of this theorem.

In particular, we obtain the following results on the existence of solutions for quasi-equilibrium problems concerning lower and upper semi-continuous multivalued mappings.

Corollary 3.2. We assume that the following conditions hold:

(i) $D, K$ are nonempty convex compact sets;

(ii) $P: D \times K \rightarrow 2^{D}$ is a continuous multivalued mapping with nonempty closed convex values;

(iii) $Q: D \times K \rightarrow 2^{K}$ is a u.s.c multivalued mapping with nonempty closed convex values;

(iv) $G: D \times K \rightarrow 2^{X}$ is a l.s.c multivalued mapping with nonempty values;

(v) For any $(x, y) \in P(x, y) \times Q(x, y), G(x, y) \subset T_{P(x, y)}(x)$.

Then there exists $(\bar{x}, \bar{y}) \in D \times K$ such that

1) $\bar{x} \in P(\bar{x}, \bar{y})$;

2) $\bar{y} \in Q(\bar{x}, \bar{y})$;

3) $0 \in G(\bar{x}, \bar{y})$.

Proof. We define the multivalued mapping $H: D \times K \rightarrow 2^{X}$ by

$$
H(x, y)=0,(y, x) \in D \times K .
$$

We can easily verify that all conditions of Theorem 3.1 are satisfied. Applying this theorem, we conclude there exists $(\bar{x}, \bar{y}) \in D \times K$ such that

1) $\bar{x} \in P(\bar{x}, \bar{y})$;

2) $\bar{y} \in Q(\bar{x}, \bar{y})$;

3) $G(\bar{x}, \bar{y}) \cap H(\bar{x}, \bar{y}) \neq \emptyset$.

This implies that $0 \in G(\bar{x}, \bar{y})$. This completes the proof.

Corollary 3.3. We assume that the following conditions hold:

(i) $D, K$ are nonempty convex compact sets;

(ii) $P: D \times K \rightarrow 2^{D}$ is a continuous multivalued mapping with nonempty closed convex values;

(iii) $Q: D \times K \rightarrow 2^{K}$ is a u.s.c multivalued mapping with nonempty closed convex values;

(iv) $H: D \times K \rightarrow 2^{X}$ is u.s.c multivalued mapping with convex and closed values;

(vi) For any $(x, y) \in P(x, y) \times Q(x, y),-H(x, y) \subset T_{P(x, y)}(x)$.

Then there exists $(\bar{x}, \bar{y}) \in D \times K$ such that

1) $\bar{x} \in P(\bar{x}, \bar{y})$;

2) $\bar{y} \in Q(\bar{x}, \bar{y})$;

3) $0 \in H(\bar{x}, \bar{y})$.

Proof. We define the multivalued mapping $G: D \times K \rightarrow 2^{X}$ by

$$
G(x, y)=0,(y, x) \in D \times K \text {. }
$$

We can easily verify that all conditions of Theorem 3.1 are satisfied. Applying this theorem we conclude there exists $(\bar{x}, \bar{y}) \in D \times K$ such that

1) $\bar{x} \in P(\bar{x}, \bar{y})$;

2) $\bar{y} \in Q(\bar{x}, \bar{y})$; 
3) $G(\bar{x}, \bar{y}) \cap H(\bar{x}, \bar{y}) \neq \emptyset$.

This implies that $0 \in H(\bar{x}, \bar{y})$. This completes the proof.

Corollary 3.4. We assume that the following conditions hold:

(i) $D, K$ are nonempty convex compact sets;

(ii) $P: D \times K \rightarrow 2^{D}$ is continuous multivalued mapping with nonempty closed convex values, $Q$ : $D \times K \rightarrow 2^{K}$ is a u.s.c multivalued mapping with nonempty closed convex values;

(iii) $F: D \times K \rightarrow 2^{D}$ is a l.s.c multivalued mapping with nonempty values;

(iv) $E: D \times K \rightarrow 2^{D}$ is a u.s.c multivalued mapping with nonempty closed convex values;

(v) $F(x, y)-E(x, y) \subset T_{P(x, y)}(x)$.

Then there exists $(\bar{x}, \bar{y}) \in D \times K$ such that

1) $\bar{x} \in P(\bar{x}, \bar{y})$;

2) $\bar{y} \in Q(\bar{x}, \bar{y})$;

3) $(F(\bar{x}, \bar{y})-\bar{x}) \cap(E(\bar{x}, \bar{y})-\bar{x}) \neq \emptyset$.

Proof. We define the multivalued mapping $G, H: D \times K \rightarrow 2^{X}$ by

$$
G(x, y)=F(x, y))-x ; H(x, y)=E(x, y)-x(y, x) \in D \times K .
$$

We can easily verify that all conditions of Theorem 3.1 are satisfied. Applying this theorem we conclude there exists $(\bar{x}, \bar{y}) \in D \times K$ such that

1) $\bar{x} \in P(\bar{x}, \bar{y})$

2) $\bar{y} \in Q(\bar{x}, \bar{y})$;

3) $G(\bar{x}, \bar{y}) \cap H(\bar{x}, \bar{y}) \neq \emptyset$.

This implies that $(F \bar{x}, \bar{y})-\bar{x}) \cap(E(\bar{x}, \bar{y})-\bar{x}) \neq \emptyset$. This completes the proof.

Remark 3.5. Corollary 3.4 unites the Ky Fan Theorem and the Browder-Ky Fan theorem together. To get the Ky Fan Theorem, we take $F(x, y)=x, x \in D$. To get Browder-Ky Fan theorem, we take $E(x, y)=x, x \in D$.

In particular, we have the fixed point following results of lower and upper semi-continuous multivalued mappings. They are generalizations of Browder - Ky Fan's Theorem and Ky Fan's theorem.

Corollary 3.6. We assume that the following conditions hold:

(i) $D$ is nonempty convex compact subset of $X$;

(ii) $F: D \rightarrow 2^{X}$ is a l.s.c mapping with nonempty values and $F(x)-x \subset T_{D}(x)$, for any $x \in D$. Then there exists $\bar{x} \in D$ such that $\bar{x} \in F(\bar{x})$.

Proof. The proof of this corollary follows immediately from Theorem 3.1 with $P(x, y)=Q(x, y)=$ $D, G(x, y)=F(x)-x, H(x, y)=0$, for all $(x, y) \in D \times K$.

Corollary 3.7. We assume that the following conditions hold:

(i) $D$ is nonempty convex compact subset of $X$;

(ii) $F: D \rightarrow 2^{X}$ is a u.s.c mapping with nonempty convex compact values and $F(x)-x \subset T_{D}(x)$, for any $x \in D$. Then there exists $\bar{x} \in D$ such that $\bar{x} \in F(\bar{x})$.

Proof. The proof of this corollary follows immediately from Theorem 3.1 with $P(x, y)=Q(x, y)=$ $D, G(x, y)=0, H(x, y)=x-F(x)$, for all $(x, y) \in D \times K$. 


\section{SOME APPLICATIONS}

Further, $D \subset X, K \subset Z$ are nonempty subsets. Given multivalued mappings $P: D \times K \rightarrow 2^{D}, Q$ : $D \times K \rightarrow 2^{K}$ and $F_{1}: K \times D \times D \times D \rightarrow 2^{Y}$, we are interested in the problem of finding $(\bar{x}, \bar{y}) \in D \times K$ such that

i) $\bar{x} \in P(\bar{x}, \bar{y})$;

ii) $\bar{y} \in Q(\bar{x}, \bar{y})$;

iii) $0 \in F_{1}(\bar{y}, \bar{x}, \bar{x}, t)$, for all $t \in P(\bar{x}, \bar{y})$.

This problem is called a general quasi-equilibrium problem of type I, denoted by $(G E P)_{I}$, in which the multivalued mappings $P, Q$ are called constraint mappings and $F_{1}$ is called a utility multivalued mapping.

Next, given multivalued mappings $P, P_{0}: D \times K \rightarrow 2^{D}, Q: D \times K \rightarrow 2^{K}, Q_{0}, D \times D \times K \rightarrow 2^{K}$ and $F_{2}: K \times K \times D \times D \rightarrow 2^{Y}$, we are interested in the problem of finding $(\bar{x}, \bar{y}) \in D \times K$ such that

$$
\bar{x} \in P(\bar{x}, \bar{y}), \bar{y} \in Q(\bar{x}, \bar{y})
$$

and

$$
0 \in F_{2}(\bar{y}, v, \bar{x}, t) \text {, for all } t \in P_{0}(\bar{x}, \bar{y}) \text { and } v \in Q_{0}(\bar{x}, t, \bar{y}) .
$$

This problem is called a general quasi-equilibrium problem of type II , denoted by $(G E P)_{I I}$, in which the multivalued mappings $P, P_{0}$ and $Q, Q_{0}$ are called constraint mappings and $F_{2}$ is called a utility multivalued mapping. The problem of finding $(\bar{x}, \bar{y}) \in D \times K$ such that

$$
\begin{gathered}
\bar{x} \in P(\bar{x}, \bar{y}), \bar{y} \in Q(\bar{x}, \bar{y}) \\
0 \in F_{1}(\bar{y}, \bar{x}, \bar{x}, t), \text { for all } t \in P(\bar{x}, \bar{y})
\end{gathered}
$$

and

$$
0 \in F_{2}(\bar{y}, v, \bar{x}, t) \text {, for all } t \in P_{0}(\bar{x}, \bar{y}) \text { and } v \in Q_{0}(\bar{x}, t, \bar{y}) .
$$

is called a mixed general quasi-equilibrium problem, in which the multivalued mappings $P, P_{0}$ and $Q, Q_{0}$ are called constraint mappings and $F_{1}, F_{2}$ are called utility multivalued mappings.

We apply the obtained results in Section 3 to get the existence to solutions for $(G E P)_{I},(G E P)_{I I}$ and for mixed general quasi-equilibrium problem of them. But, we first prove some propositions to be used later.

Proposition 4.1. We assume that the following conditions are sufficient:

i) $D$ and $K$ are nonempty convex compact subsets;

ii) $P: D \times K \rightarrow 2^{D}$ is a continuous multivalued mapping with nonempty closed values;

iii) $Q: D \times K \rightarrow 2^{K}$ is a u.s.c multivalued mapping with nonempty closed values;

iv) The set $A=\left\{(y, x, z, t) \in K \times D \times D \times D \mid 0 \in F_{1}(y, x, z, t)\right\}$ is closed ;

v) For any fixed $(y, x) \in K \times D$, the set

$$
B=\left\{z \in P(x, y) \mid 0 \in F_{1}(y, x, z, t) \text { for all } t \in Q(x, y)\right\}
$$

is nonempty convex.

Then, the multivalued mapping $F: D \times K \rightarrow 2^{D}$ by

$$
F(x, y)=\left\{z \in P(x, y) \mid 0 \in F_{1}(y, x, z, t), \text { for all } t \in P(x, y)\right\}
$$

is u.s.c with nonempty convex closed values on $D \times K$. 
Proof. Condition v) implies that $F(x, y) \neq \emptyset$ and nonempty convex for any $(x, y) \in D \times K$. Now, we show that $F$ is u.s.c on $D \times K$. Since $D$ is compact and $F$ has closed values, therefore, we need only to prove that $F$ is a closed mapping. Indeed, let $\left(x_{\alpha}, y_{\alpha}\right)$ be a net converging to $(x, y)$ and $z_{\alpha}$ be a net with $z_{\alpha} \in F\left(x_{\alpha}, y_{\alpha}\right), z_{\alpha} \rightarrow z$. We have to show $z \in F(x, y)$. Indeed, we can see $0 \in F_{1}\left(y_{\alpha}, x_{\alpha}, z_{\alpha}, t_{\alpha}\right)$, $\forall t_{\alpha} \in P\left(x_{\alpha}, y_{\alpha}\right)$. Let $v \in P(x, y)$ be arbitrary. Since $P$ is l.s.c, there is $t_{\alpha} \in P\left(x_{\alpha}, y_{\alpha}\right), t_{\alpha} \rightarrow t$. Hence, we get $0 \in F_{1}\left(y_{\alpha}, x_{\alpha}, z_{\alpha}, t_{\alpha}\right)$. For $\left(y_{\alpha}, x_{\alpha}, z_{\alpha}, t_{\alpha}\right) \rightarrow(y, x, z, t)$ and the set $A$ is closed, we deduce $(y, x, z, t) \in A$. Hence, $0 \in F_{1}(y, x, z, t)$, for all $t \in P(x, y)$. This shows that the multivalued mapping $F$ is closed, and then $F$ is u.s.c on $D \times K$. Thus, the proof of the proposition is completed.

The following corollary on the existence to solutions of $(G E P)_{I}$ generalizes Theorems 3.1 in [7].

Corollary 4.2. $(G E P)_{I}$ has a solution if $D, K, P, Q, F_{1}$ and $F$ satisfy all conditions of Proposition 4.1.

Proof. It follows immediately from Corollary 3.3 with $H(x, y)=x-F(x, y)$ that there exists $(\bar{x}, \bar{y}) \in$ $D \times K$ such that

1) $\bar{x} \in P(\bar{x}, \bar{y})$;

2) $\bar{y} \in Q(\bar{x}, \bar{y})$;

3) $\bar{x} \in F(\bar{x}, \bar{y})$.

This gives $0 \in F_{1}(\bar{y}, \bar{x}, \bar{x}, t), \forall t \in P(\bar{x}, \bar{y})$.

We have the following proposition.

Proposition 4.3. We assume that the following conditions are satisfied.

i) D is a nonempty convex compact subset;

ii) $P: D \times K \rightarrow 2^{D}$ is a multivalued mapping with a nonempty closed fixed set $B=\{(x, y) \in D \times$ $K \mid x \in P(x, y)), y \in Q(x, y)\}$;

iii) $P_{0}: D \times K \rightarrow 2^{D}$ is a multivalued mapping having open lower sections and co $\left(P_{0}(x, y)\right)$ is contained in $P(x, y)$ for each $(x, y) \in D \times K$;

iv) For any fixed $t \in D$, the set

$$
A=\left\{(x, y) \in D \times K \mid 0 \notin F_{2}(y, v, x, t), \text { for some } v \in Q_{0}(x, t, y)\right\}
$$

is open in $D$.

Then, the multivalued mapping $G: D \times K \rightarrow 2^{D}$ defined by

$$
G(x, y)=\left\{t \in P_{0}(x, y) \mid 0 \notin F_{2}(y, v, x, t), \text { for some } v \in Q_{0}(x, t, y)\right\},
$$

$(x, y) \in D \times K$, is l.s.c on $D \times K$.

Proof. We can write

$$
G(x, y)=\left\{t \in D \mid 0 \notin F_{2}(y, v, x, t), \text { for some } v \in Q_{0}(x, t, y)\right\} \cap P_{0}(x, y) .
$$

For any $t \in D, G^{-1}(t)$ is the set

$$
\begin{gathered}
\left\{(x, y) \in D \times K \mid 0 \notin F_{2}(y, v, x, t), \text { for some } v \in Q_{0}(x, t, y)\right\} \cap P_{0}^{-1}(t) \\
=A \cap P_{0}^{-1}(t),
\end{gathered}
$$

is open. Hence the, multivalued mapping $G$ has open lower sections. This follows that $G$ is 1.s.c. The proof is complete. 
Now, we assume that for any fixed $(x, y) \in D \times K$ the multivalued mapping $Q_{0}(x, ., y): D \rightarrow 2^{K}$ is 1.s.c and the multivalued mapping $F_{2}(., ., t): D \times K \rightarrow 2^{Y}$ is closed.

Proposition 4.4. We assume that the following conditions are satisfied.

i) D is a nonempty convex compact subset;

ii) $P: D \times K \rightarrow 2^{D}$ is a multivalued mapping with a nonempty closed fixed set $B=\{(x, y) \in D \times$ $K \mid x \in P(x, y), y \in Q(x, y)\}$

iii) $P_{0}: D \times K \rightarrow 2^{D}$ is a multivalued mapping having open lower sections and $P_{0}(x, y)$ is contained in $P(x, y)$ for each $(x, y) \in D \times K$;

iv) For any fixed $t \in D$, the multivalued mapping $Q_{0}(., t,):. D \times K \rightarrow 2^{K}$ is l.s.c and the multivalued mapping $F_{2}(., ., ., t): K \times K \times D \rightarrow 2^{Y}$ is closed.

Then, the multivalued mapping $G: D \times K \rightarrow 2^{D}$ defined by

$$
G(x, y)=\left\{t \in P_{0}(x, y) \mid 0 \notin F_{2}(y, v, x, t), \text { for some } v \in Q_{0}(x, t, y)\right\},
$$

$(x, y) \in D \times K$, is l.s.c on $D \times K$.

Proof. For given $t \in D$, we set

$$
C=\left\{(x, y) \in D \times K \mid 0 \in F_{2}(y, v, x, t), \text { for all } v \in Q_{0}(x, t, y)\right\}
$$

and we show that this set is closed in $D \times K$. Indeed, let $\left(x_{\alpha}, y_{\alpha}\right)$ be a net in $C$ such that $x_{\alpha} \rightarrow x, y_{\alpha} \rightarrow y$. Taking $v \in Q_{0}(x, t, y)$ arbitrary, $Q_{0}(., t,$.$) is l.s.c at (x, y)$. We can find $v_{\alpha} \in Q_{0}\left(x_{\alpha}, t, y_{\alpha}\right)$ such that $v_{\alpha} \rightarrow v$. Since $\left(x_{\alpha}, y_{\alpha}\right) \in C$, we have $0 \in F_{2}\left(y_{\alpha}, v_{\alpha}, x_{\alpha}, t\right)$. The closedness of $F_{2}(., ., ., t)$ follows $0 \in F_{2}(y, v, x, t)$. Therefore, $0 \in F_{2}(y, v, x, t)$ for all $v \in Q_{0}(x, t, y)$. So $(x, y) \in C$, which shows that $C$ is a closed set. This implies that the set

$$
A=D \backslash C=\left\{(x, y) \in D \times K \mid 0 \notin F_{2}(y, v, x, t) \text {, for some } v \in Q_{0}(x, t, y)\right\}
$$

is open in $D$. Thus, Condition (iv) of Proposition 4.3 is satisfied. Therefore, to complete the proof of the corollary, it remains to apply Proposition 4.3.

The following corollary extends Theorem 2.1 in [8].

Corollary 4.5. $(G E P)_{I I}$ has a solution if $D, K, P, P_{0}, Q, Q_{0}$, and $F_{2}$ satisfy all conditions of Proposition 4.3 or Proposition 4.4 and $0 \in F_{2}(y, v, x, x)$ for any $y \in K$ and $v \in Q_{0}(x, x, y)$.

Proof. It follows immediately from Corollary 3.2 for $\tilde{G}(x, y)=G(x, y)-x$, where $G$ is defined by Proposition 4.3 or Proposition 4.4, that $\tilde{G}$ is 1.s.c on $D \times K$. If for any $(x, y) \in D \times K$ such that $x \in$ $P(x, y), y \in Q(x, y)$ and $\tilde{G}(x, y) \neq \emptyset$, then there exists $\left(x^{*}, y^{*}\right) \in D \times K$ such that $0 \in \tilde{G}\left(x^{*}, y^{*}\right)$. This follows $x^{*} \in G\left(x^{*}, y^{*}\right)$. Hence, $x^{*} \in P_{0}\left(x^{*}, y^{*}\right)$ and $0 \notin F_{2}\left(y^{*}, v, x^{*}, x^{*}\right)$, for some $v \in Q_{0}\left(x^{*}, x^{*}, y^{*}\right)$. We have a contradiction. Thus, we conclude that there exists $(\bar{x}, \bar{y}) \in D \times K$ such that

1) $\bar{x} \in P(\bar{x}, \bar{y})$;

2) $\bar{y} \in Q(\bar{x}, \bar{y})$;

3) $G(\bar{x}, \bar{y})=\emptyset$.

This gives $0 \in F_{2}(\bar{y}, v, \bar{x}, t)$, for all $t \in P_{0}(\bar{x}, \bar{y}), v \in Q_{0}(\bar{x}, t, \bar{y})$.

Next, we apply Corollary 3.6 to prove the existence of solutions to mixed general quasi-equilibrium problems of $(G E P)_{I}$ and $(G E P)_{I I}$. The following theorem is a generalization of Theorem 3.1 in [12]. 
Theorem 4.6. We assume that he following conditions are sufficient.

i) $D$ and $K$ are nonempty convex compact subsets;

ii) $P: D \times K \rightarrow 2^{D}$ is a continuous multivalued mapping with nonempty convex closed values;

iii) $Q: D \times K \rightarrow 2^{K}$ is a u.s.c multivalued mapping with nonempty convex closed values;

iv) The set $A=\left\{(y, x, z, t) \in K \times D \times D \times D \mid 0 \in F_{1}(y, x, z, t)\right\}$ is closed;

v) For any fixed $(y, x) \in K \times D$, the set

$$
B=\left\{z \in P(x, y) \mid 0 \in F_{1}(y, x, z, t) \text { for all } t \in Q(x, y)\right\}
$$

is nonempty convex;

vi) $P_{0}: D \times K \rightarrow 2^{D}$ is a multivalued mapping having open lower sections and for each $(x, y) \in$ $P_{0}(x, y) \times Q(x, y)$, one has

$$
0 \in F_{1}(y, x, x, t), \text { for all } t \in P(x, y) ;
$$

vii) $Q_{0}: D \times D \times K \rightarrow 2^{K}, F_{2}: K \times K \times D \times D \rightarrow 2^{Y}$ are multivalued mapping such that for any fixed $t \in D$, the set

$$
C=\left\{(x, y) \in D \times K \mid 0 \notin F_{2}(y, v, x, t), \text { for some } v \in Q_{0}(x, t, y)\right\}
$$

is open in $D$.

viii) $0 \in F_{2}(y, v, x, x)$ for all $(x, y) \in P(x, y) \times Q(x, y), v \in Q_{0}(x, x, y)$.

Then there exists $(\bar{x}, \bar{y}) \in D \times K$ such that

1) $\bar{x} \in P(\bar{x}, \bar{y})$;

2) $\bar{y} \in Q(\bar{x}, \bar{y})$;

3) $0 \in F_{1}(\bar{y}, \bar{x}, \bar{x}, t)$, for all $t \in P(\bar{x}, \bar{y})$.

4) $0 \in F_{2}(\bar{y}, v, \bar{x}, t)$, for all $t \in P_{0}(\bar{x}, \bar{y})$ and $v \in Q_{0}(\bar{x}, t, \bar{y})$.

Proof. We define the multivalued mappings $H, G: D \times K \rightarrow 2^{D}$ by

$$
\begin{gathered}
H(x, y)=\left\{z \in P(x, y) \mid 0 \in F_{1}(y, x, z, t), \text { for all } t \in P(x, y)\right\}, \\
G(x, y)=\left\{t \in P_{0}(x, y) \mid 0 \notin F_{2}(y, v, x, t), \text { for some } v \in Q_{0}(x, t, y)\right\},
\end{gathered}
$$

$(x, y) \in D \times K$. Proposition 4.1 shows that $H$ is u.s.c with nonempty convex closed values and $G$ is 1. s.c on $D \times K$. Observe that if for some $(\bar{x}, \bar{y}) \in H(\bar{x}, \bar{y}) \times Q(\bar{x}, \bar{y})$, it gives $G(\bar{x}, \bar{y}) \cap P_{0}(\bar{x}, \bar{y})=\emptyset$, then

i) $\bar{x} \in P(\bar{x}, \bar{y})$;

ii) $\bar{y} \in Q(\bar{x}, \bar{y})$;

iii) $0 \in F_{1}(\bar{y}, \bar{x}, \bar{x}, t)$, for all $t \in P(\bar{x}, \bar{y})$.

and

$$
0 \in F_{2}(\bar{y}, v, \bar{x}, t), \text { for all } t \in P_{0}(\bar{x}, \bar{y}) \text { and } v \in Q_{0}(\bar{x}, t, \bar{y}),
$$

and hence the proof of the theorem is completed. Thus, our aim is to show the existence of such a point $(\bar{x}, \bar{y})$. Indeed, by contrary, for any $(x, y) \in H(x, y) \times Q(x, y), G(x, y) \cap P_{0}(x, y) \neq \emptyset$. We consider the multivalued mapping $T: D \times K \rightarrow 2^{D \times K}$ defined by

$$
T(x, y)= \begin{cases}G(x, y) \cap P_{0}(x, y) \times\{y\}, & \text { if } x \in H(x, y), \\ P_{0}(x, y) \times\{y\}, & \text { else. }\end{cases}
$$


We show that $T$ verifies the hypotheses of Theorem 1.2 of Section 1. Apply this corollary, we conclude that there is a point $(\bar{x}, \bar{y}) \in D \times K$ such that $\bar{x} \in P(\bar{x}, \bar{y}), \bar{y} \in Q(\bar{x}, \bar{y})$ and $(\bar{x}, \bar{y}) \in T(\bar{x}, \bar{y})$. If $\bar{x} \in H(\bar{x}, \bar{y})$, then $\bar{x} \in G(\bar{x}, \bar{y}) \cap P_{0}(\bar{x}, \bar{y})$. Hence $0 \notin F_{2}(\bar{y}, v, \bar{x}, \bar{x})$, for some $v \in Q_{0}(\bar{x}, \bar{x}, \bar{y})$, we have a contradiction to Condition viii). If $\bar{x} \notin H(\bar{x}, \bar{y})$, then $(\bar{x}, \bar{y}) \in T(\bar{x}, \bar{y})=P_{0}(\bar{x}, \bar{y}) \times\{\bar{y}\}$. By Condition vi), $\bar{x} \in H(\bar{x}, \bar{y})$. We also have a contradiction. The proof of the theorem is complete.

Remark 4.7. We can understand Theorem 4.6 as solving $(G E P)_{I I}$ on the set of solutions of the $(G E P)_{I}$.

In particular, for scalar case, we consider the functions: $\phi_{1}: K \times D \times D \times D \rightarrow R, \phi_{2}: K \times K \times D \times D \rightarrow$ $R$.

Corollary 4.8. We assume that the following conditions hold:

i) $D$ and $K$ are nonempty convex compact subsets;

ii) $P: D \times K \rightarrow 2^{D}$ is a continuous multivalued mapping with nonempty convex closed values;

iii) $Q: D \times K \rightarrow 2^{K}$ is a u.s.c multivalued mapping with nonempty convex closed values;

iv) The set $A=\left\{(y, x, z, t) \in K \times D \times D \times D \mid \phi_{1}(y, x, z, t) \leq 0\right\}$ is closed ;

v) For any fixed $(y, x) \in K \times D$, the set $B=\left\{z \in P(x, y) \mid \phi_{1}(y, x, z, t) \leq 0\right.$ for all $\left.t \in Q(x, y)\right\}$ is nonempty and convex;

vi) $P_{0}: D \times K \rightarrow 2^{D}$ is a multivalued mapping having open lower sections and for each $(x, y) \in$ $P_{0}(x, y) \times Q(x, y)$, one has $\phi_{1}(y, x, x, t) \geq 0$, for all $t \in P(x, y)$;

vii) $Q_{0}: D \times D \times K \rightarrow 2^{K}, \phi_{2}: K \times K \times D \times D \rightarrow 2^{Y}$ are mappings such that for any fixed $t \in D$, the set

$$
C=\left\{(x, y) \in D \times K \mid \phi_{2}(y, v, x, t)<0, \text { for some } v \in Q_{0}(x, t, y)\right\}
$$

is open in $D$.

viii) $\phi_{2}(y, v, x, x) \geq 0$ for all $\left.(x, y) \in P(x, y) \times Q x, y\right), v \in Q_{0}(x, x, y)$.

Then there exists $(\bar{x}, \bar{y}) \in D \times K$ such that

1) $\bar{x} \in P(\bar{x}, \bar{y})$;

2) $\bar{y} \in Q(\bar{x}, \bar{y})$;

3) $\phi_{1}(\bar{y}, \bar{x}, \bar{x}, t) \leq 0, \quad \forall t \in P(\bar{x}, \bar{y})$.

4) $\phi_{2}(\bar{y}, v, \bar{x}, t) \geq 0, \quad \forall t \in P_{0}(\bar{x}, \bar{y})$ and $v \in Q_{0}(\bar{x}, t, \bar{y})$.

Proof. We define mappings $F_{1}: K \times D \times D \times D \rightarrow 2^{R}$ and $F: K \times K \times D \times D \rightarrow 2^{R}$ defined by

$$
\begin{aligned}
& F_{1}(y, x, z, t)=\phi_{1}(y, x, z, t)+R_{+} \\
& F_{2}(y, v, x, t)=\phi(y, v, x, t)-R_{+} .
\end{aligned}
$$

We see that the set

$$
A=\left\{(y, x, z, t) \in K \times D \times D \times D \mid 0 \in F_{1}(y, x, z, t)\right\}=\left\{(y, x, z, t) \in K \times D \times D \times D \mid \phi_{1}(y, x, z, t) \leq 0\right\}
$$

is closed and the set

$$
\begin{gathered}
B=\left\{z \in P(x, y) \mid 0 \in \phi_{1}(y, x, z, t) \leq 0 \text { for all } t \in Q(x, y)\right\} \\
=\left\{z \in P(x, y) \mid 0 \in F_{1}(y, x, z, t) \text { for all } t \in Q(x, y)\right\}
\end{gathered}
$$


is nonempty and convex. $(x, y) \in P_{0}(x, y) \times Q(x, y), 0 \in F_{1}(y, x, x, t)$, for all $t \in P(x, y)$ if and only if $(x, y) \in P_{0}(x, y) \times Q(x, y)$. One has $\phi_{1}(y, x, x, t) \leq 0$, for all $t \in P(x, y)$. Further, the set

$$
\begin{aligned}
C & =\left\{(x, y) \in D \times K \mid 0 \notin F_{2}(y, v, x, t), \text { for some } v \in Q_{0}(y, x, t)\right\} \\
& =\left\{(x, y) \in D \times K \mid \phi_{2}(y, v, x, t)<0, \text { for some } v \in Q(x, y)\right\}
\end{aligned}
$$

is open in $D \times K$ and $\phi_{2}(y, v, x, x) \geq 0$ for all $\left.(x, y) \in P(x, y) \times Q x, y\right), v \in Q_{0}(x, x, y)$ implies that $0 \in$ $F_{2}(y, v, x, x)$ for all $\left.(x, y) \in P(x, y) \times Q x, y\right), v \in Q_{0}(x, x, y)$. Therefore, to complete the proof of the corollary, it remains to apply Theorem 4.6.

It implies from the following remark that Corollary 4.8 generalizes Ky Fan's result in [13] and Minty's result in [14].

Remark 4.9. 1) In the case that $\phi_{1}$ is lower semi-continuous function and for any fixed $(y, x, t) \in K \times$ $D \times D$ the function $\phi_{1}(y, x, ., t): D \rightarrow R$ is quasi-convex function, Conditions vi) and v) are satisfied.

2) In the case that $Q_{0}: D \times D \times K \rightarrow 2^{K}$ is lower semicontinuous mapping and $\phi_{2}: K \times K \times D \times D \rightarrow 2^{Y}$ is a function such that for any fixed $t \in D$, the function $\phi_{2}(., ., ., t): K \times D \times D \rightarrow R$ is upper semi-continuous, Condition vii) is satisfied.

\section{Acknowledgements}

This work was supported by the NAFOSTED of Viet Nam under the decision Nr.30/QD-HDQL-NAFOSTED, Code 101.02-2016.11. The authors would like to thank the anonymous referees for some useful suggestions which improved the quality of the paper.

\section{REFERENCES}

[1] N.C. Yannelis, N.D. Prabhakar, Existence of maximal elements and equilibria in linear topological spaces, J. Math. Economics, 12 (1983), 233-245.

[2] H. Ben-El-Mechaiekh, Fixed points for compact set-valued maps, Quest. Answers Gen. Topol. 10 (1992), 153-156.

[3] C. D. Horvath, Extension and selection theorems in topological spaces with a generalized convexity structure, Ann. Fac. Sci. Toulouse 2 (1993), 253-269.

[4] X. Wu, A new fixed point theorem and its applications, Proc. Amer. Math. Soc. 125 (1997), 1779-1783.

[5] S. Park, Continuous selection theorems in generalized convex spaces, Numer. Funct. Anal. Optim. 25 (1999), $567-583$.

[6] N.X. Tan, N.Q. Hoa, Quasi-equilibrium problems and fixed point theorems of 1.s.c mappings, Adv. Nonlinear Var. Inequal. 19 (2016), 52-63.

[7] T.T.T. Duong, N.X. Tan, On the existence of solutions to generalized quasi-equilibrium problems of type I and related problems, Adv. Nonlinear Var. Inequal. 13 (2010), 29-47.

[8] T.T.T. Duong, N.X. Tan, On the existence of solutions to generalized quasi-equilibrium, J. Global Optim. 52 (2012), 711-728.

[9] K. Fan, Fixed-point and minimax theorems in locally convex topological linear spaces, Proc. Natl. Acad. Sci. USA 38 (1952), 121-126.

[10] N.X. Tan, On the existence of solutions of quasi-variational inclusion problems, J. Optim. Theory Appl. 123 (2004), 619-638.

[11] W. Rudin, Principles of Mathematical Analysis, Third Edition, McGraw-hill, 1964.

[12] T.T.T Duong, Mixed generalized quai-equilibrium problems, J. Global Optim. 56 (2013), 647-667.

[13] K. Fan, A minimax inequality and application in inequality iii, pp 103-113, Academic Press, New York, 1972.

[14] G. J. Minty, On the generalization of direct method of the calculus of variations, Bull. Amer. Math. Soc. 73 (1967), 314-321. 